\title{
Idempotency of Left-Sided Identity on Singular Transformation Semigroup
}

\author{
Adeniji, A. O. \\ Department of Mathematics, Faculty of Science, University of Abuja, Abuja.
}

\begin{abstract}
Idempotent elements are left identity in the Semigroup generated by the left action of each singular element on Symmetric group.
\end{abstract}

Keywords: Left Identity; Idempotent; Symmetric Group; Singular Semigroup.

\section{Introduction}

Let $\mathrm{X}$ be a finite set of elements following the natural ordering of numbers. The notation $T_{n}$ is the full transformation semigroup, $S_{n}$ is the symmetric group and $T_{n} \backslash S_{n}$ is the singular transformation semigroup which can as well be denoted by $\operatorname{Sing}_{\mathrm{n}}$, [4]. Let $\mathrm{S}$ be a semigroup generated by the relation aG where a $\in T_{n} \backslash S_{n}$ and $G$ $=\mathrm{S}_{\mathrm{n}}$.

In 1968, Tainiter [6] came up with a formula for knowing the number of idempotent in $\mathrm{T}_{\mathrm{n}}$, which he denoted by $\mathrm{H}_{\mathrm{n}}$ with $\mathrm{H}_{\mathrm{n}}=\sum_{p=1}^{n}\left(\begin{array}{l}n \\ p\end{array}\right) p^{n-p}$. Araújo and Cameron [1] obtained that a semigroup $\mathrm{S}$ generated by the conjugates $\mathrm{g}^{-1}$ ag with a $\in \mathrm{T}_{\mathrm{n}} \backslash \mathrm{S}_{\mathrm{n}}$ and $\mathrm{g} \in \mathrm{S}_{\mathrm{n}}$, is idempotent generated, regular and that $\mathrm{S}=\left\langle a, S_{n}\right\rangle \backslash S_{n}$.

A partition of a set $\mathrm{S}$ is a pairwise disjoint set of non-empty subsets, called "parts" or "blocks" or "cells", whose union is all of $S$. The semigroup $S=T_{n} \backslash S_{n}$ as studied in this work, is decomposable into non - overlapping cells using the semigroup $\mathrm{aS}_{\mathrm{n}}$.

Howie [4] used Sing $\mathrm{n}_{\mathrm{n}}$ to denote singular mappings and proved that $\operatorname{Sing}_{\mathrm{n}}$, the semigroup of all singular mappings of $X=\{1,2, \ldots n\}$ into itself, is generated by its idempotents of defect 1 . It was also proved that if $\mathrm{n} \geq 3$ then a minimal generating set for $\operatorname{Sing}_{\mathrm{n}}$ contains $\frac{n(n+1)}{2}$ transformations of defect 1 [3]. In his work, Winbush [7] showed as Theorem 1.1 that a semigroup has at most one identity element and because of that Theorem, exactly one of the following statements must hold for a semigroup $S$ that is a monoid:

(i) S has no left and no right identity element;

(ii) $\mathrm{S}$ has one or more left identity elements, but no right identity element;

(iii) $\mathrm{S}$ has one or more right identity elements, but no left identity element;

(iv) $\mathrm{S}$ has a unique two-sided identity, and no other right or left identity element.

In consonant with Wimbush work [7], it is seen in this paper that a semigroup that has left-sided identity or right-sided identity element does not have the identity, hence not a monoid.

Let $\alpha \in \operatorname{Sing}_{n}$. The kernel of $\alpha$ is defined as $\operatorname{Ker}(\alpha)=\left\{(\mathrm{x}, \mathrm{y}) \in \mathrm{X}_{\mathrm{n}} \mathrm{X} \mathrm{X} \_\mathrm{n}: \mathrm{x} \alpha=\mathrm{y} \alpha\right\}$. The semigroup, Sing $_{\mathrm{n}}$ can be partitioned using the kernel of its elements.

In addition, Howie [5] explained period and index of an element as follows:

Let a be an element of a semigroup $\mathrm{S}$ and consider the monogenic semigroup

$\langle a\rangle=\left\{a, a^{2}, a^{3}, \ldots\right\}$ generated by a. If there are no repetitions in the list $a, a^{2}, a^{3}, \ldots \$$ that is,

$a^{m}=a^{n} \Rightarrow m=n$, then evidently $(\langle a\rangle, \cdot)$ is isomorphic to the semigroup $(\mathrm{N},+)$ of natural numbers with

respect to addition. In such a case we say that a is an infinite order in S. Suppose now that there are repetitions among the powers of a. Then the set

$\left\{x \in N:(\exists y \in N) a^{x}=a^{y}, x \neq y\right\}$

is non-empty and so has a least element. Let us denote this least element by $\mathrm{m}$ and call it the index of the element of a. Then the set

$\left\{x \in N: a^{x+m}=a^{m}\right\}$ 
is non-empty and so it too has a least element $r$, which is called the period of a. $\mathrm{m}$ and $\mathrm{r}$ are respectively referred to as the index and period of the monogenic semigroup $\langle a\rangle$. Let a be an element with index $\mathrm{m}$ and period $\mathrm{r}$. Thus, $a^{\mathrm{m}}=\mathrm{a}^{\mathrm{m}+\mathrm{r}}$.

\section{Results}

The following results are on the semigroup of singular transformations denoted by S.

\section{Theorem 1.}

The number of partitions of the semigroup $T_{n} \mid S_{n}$ using a subsemigroup $a S_{n}$ for a $\in T_{n} \mid S_{n}$ is the number $f(n)=f(n-1)+f(n-3)+1$, where $f(1)=f(2)=1$ and $f(3)=2$.

Proof:

The elements of $a S_{n}$ is a subset of the singular transformation semigroup $T_{n} \backslash S_{n}$. It was known that the cardinality of $T_{n}$ is $n^{n}$ and that of $S_{n}$ is $n$ ! Elements of the same structure belong to the same cell. It was obtained in this work that the number of cells, the structure of which is determined by a, is $f(n)$ as seen in table 1.The initial values are set for $n=1,2$ and 3, through which other values of $f(n)$ are determined for $n \geq 4$. The semigroup Sing $_{\mathrm{n}}$ is thus partitioned using its kernel.

Table 1:Number of Decomposition of the Structure of Singular Transformations without Repetitions.

\begin{tabular}{|l|l|l|l|l|l|l|l|}
\hline $\mathrm{N}$ & 1 & 2 & 3 & 4 & 5 & 6 & 7 \\
\hline $\mathrm{F}(\mathrm{n})$ & 1 & 1 & 2 & 4 & 6 & 9 & 14 \\
\hline
\end{tabular}

The idempotent elements in the semigroup $S$ generated by $a S_{n}$, a $\in T_{n} \backslash S_{n}$ are identified to be left - sided identities. $\operatorname{Im}(\alpha)$ denotes image of $\alpha, \mathrm{e}_{\mathrm{L}}$ is left identity element and $|\operatorname{Im}(\alpha)|$ is the length of image of $\alpha$ in this work.

Lemma 1:

Each subsemigroup has at least two idempotent elements.

Proof:

Let the idempotent elements be $\mathrm{E}(\mathrm{S})$. The maximum length of image of $\mathrm{S}$ is $\mathrm{n}-1$. If the number of fixes in an element is $\mathrm{n}-1$ then that element is an idempotent. $\square$

Lemma 2:

The idempotents, $\mathrm{E}(\mathrm{S})$ is equivalent to the left identity element, $\mathrm{e}_{\mathrm{L}}$.

Proof:

Let $\mathrm{S}$ be a non - empty set and $\mathrm{a}, \mathrm{b}, \mathrm{e}_{\mathrm{L}} \in \mathrm{S}$. Define multiplication operation * with composition of mapping for every element $\mathrm{b} \in \mathrm{S}$, there are some elements $\mathrm{a}$ and $\mathrm{e}_{\mathrm{L}}$ such that $\mathrm{a} * \mathrm{~b}=\mathrm{b}$ and $\mathrm{e}_{\mathrm{L}} * \mathrm{~b}=\mathrm{b}$. It is immediate that $*$ is associative. If $e_{L}^{2}=\mathrm{e}_{\mathrm{L}}$ and $\mathrm{a}^{2}=\mathrm{a}$ then a and $\mathrm{e}_{\mathrm{L}}$ are left identities.

\section{Theorem 2:}

Let $\mathrm{i} \in \operatorname{Im}(\alpha)$ of the idempotents, then there are some elements $\tau$ such that $\tau \subseteq \mathrm{S}$ and $\alpha \in \tau \Rightarrow \alpha^{2}=e_{L}$. That is, $\alpha$ is the inverse of itself implying that $\mathrm{e}_{\mathrm{L}}$ is both a left and right identity.

Proof:

Lemma 2 showed that $\mathrm{E}(\mathrm{S})=\mathrm{e}_{\mathrm{L}}$ in the semigroup S. Let $\alpha \in \mathrm{S}$ such that $\alpha^{2}=\alpha=\mathrm{e}_{\mathrm{L}}$.

$\Rightarrow \alpha=e_{L}$

$\alpha e_{L}=e_{L}^{2}=e_{L}$

$\Rightarrow \alpha=1$ (identity by right cancellation law).

Also if (i) is written as

$e_{L} \alpha=e_{L} \cdot e_{L}=e_{L}$

$\Rightarrow \alpha=1$ (Identity by left cancellation law).

Hence, $\alpha$ is the inverse of itself.

Each of the partitions obtained in theorem 1 above together with elements whose images are of length one, is a subsemigroup. This subsemigroup is then defined as $S=\operatorname{ker}(\alpha) \cup|\operatorname{Im}(\alpha)|=1$.

It was established that idempotent elements are left identity in the semigroups under this study. The following results emerged from the relationship between index (i), period (p), left identity and idempotent elements.

\section{Proposition 1:}

The index (i) of $\alpha$ is one while its period (p) is $\mathrm{n}-1$ if for some t, $\alpha^{t}=\mathrm{e}$, where e is an idempotent element.

Proof: 
Let $\alpha \in \mathrm{S}$. It follows from composition of mapping that there exist $\mathrm{t}$ for some elements other than the idempotents such that

$\alpha^{t}=\mathrm{e}$.

It was shown in lemma (2) that $\mathrm{e}=\mathrm{e}_{\mathrm{L}}$, then

$\alpha^{t} \cdot \alpha=\alpha$,

$\alpha^{t+1}=\alpha^{n}=\alpha$,

Hence, $\mathrm{t}=\mathrm{n}-1$.

Proposition 2:

The idempotent generated by the monogenic subsemigroup $\langle a\rangle$ of $\operatorname{Sing}_{\mathrm{n}}$ such that $\mathrm{p}<\mathrm{i}$, is the identity element of that subsemigroup and such an idempotent is the idempotent in the kernel of the generator.

Proof:

Let $\langle a\rangle$ be a monogenic subsemigroup. There exist some $k \in N$ such that $\mathrm{a}^{\mathrm{k}} \cdot \mathrm{a}^{\mathrm{k}}=\mathrm{a}^{\mathrm{k}}$. Let

$\langle a\rangle=\left\{a, a^{2}, a^{3}, \ldots\right\}$. Composition of mapping shows that for elements $\mathrm{a}^{\mathrm{q}} \in\langle a\rangle, \mathrm{a}^{\mathrm{q}} \cdot \mathrm{a}^{\mathrm{k}}=\mathrm{a}^{\mathrm{k}} \cdot \mathrm{a}^{\mathrm{q}}$ $=a^{\mathrm{q}}$. This simply means that, the idempotent generated by an element that commutes with its generator to yield itself or another element cannot be an identity of that subsemigroup, which is any other idempotent outside the kernel of the generator, is not an identity.Any idempotent that does not have the same kernel with its generator could not be an identity. In addition, the subsemigroup $\langle a\rangle=a, a^{2}, a^{3}, \ldots$ must generate an idempotent. The following theorem clarifies this statement better.

\section{Theorem 3:}

Any idempotent generated by an element of the same kernel is an identity element; otherwise, it has a different kernel.

Proof:

Let $\alpha \in \mathrm{S}$ and an idempotent element $\beta \in S$ such that $\alpha$ and $\beta$ have the same kernel structure. If for some positive integer $\mathrm{n}, \alpha^{n}=\beta$ then $\beta^{2}=\beta$. Following the proof of Proposition $2, \beta$ is an identity element.

\section{Lemma 3:}

A set of elements generated by an element not necessarily generating itself forms a commutative subsemigroup. Proof:

Let $S=a, b, b^{2}, \ldots$ be a subsemigroup generated by a but a cannot generate itself. From composition of mappings, $S$ can be shown to be commutative since $a \cdot b=b . a, b \cdot b^{2}=b^{2} . b, \ldots$ The idempotents generated here do not belong to the same kernel with the generator.

\section{Lemma 4:}

A set of elements from a generator that generates itself is a commutative subsemigroup and hence a group.

Proof:

The proof is as in Lemma 3 and the generator generates itself. The idempotent generated is the identity (this follows from theorem 3) and each element has its inverse. In addition, all the elements generated here have the same kernel and the idempotent generated occupy the right diagonal of its Cayley's table with the elements occupying the left diagonal. $\square$

\section{Conclusion}

The semigroup of singular transformations can be partitioned using its kernels and a semigroup $\mathrm{aS}_{\mathrm{n}}$. Idempotent elements can be used as left-identity elements and as identity element if generated by an element of the same kernel structure.

\section{References}

[1]. Araújo, Joao. and Cameron, J. Peter (2013) : Permutation Groups and Transformation Semigroups: Results and Problems, arxiv.org/pdf/1308.3585.pdf, 25, 1-15.

[2]. Gonca Ayik, Hayrullah Ayik, Leyla Bugay and Osman Kelekci (2012): Generating Sets of Finite Singular Transformation Semigroups, Semigroup Forum, Research Article Dedicated to the Memory of John Mackintosh Howie (1936-2011), DOI 10.1007/s00233 - 012 - 9397 - 1, Springer Science + Business Media, LLC 2012.

[3]. Gomes, G.M.S. and Howie, J.M. (1987): On the Ranks of Certain Finite Semigroups of Transformations, Math. Proc. Camb. Philos. Soc. 101. 395-403.

[4]. Howie, J. M. (1966): The Subsemigroup Generated by the Idempotents of a Full Transformation Semigroup, Journal of London Math. Soc., (41), 707-716.

[5]. Howie, J. M. (1995): Fundamentals of Semigroup Theory, Oxford University Press New York.

[6]. Tainiter, M. (1968): A Characterization of Idempotents in Semigroups, Journal of Combinatorial Theory, (5), 370-373.

[7]. Wimbush, G. W. (1969): Algebraic Structure of Semigroups, ETD Collection for AUC Robert W. Woodruff Library. Paper 1078 